\title{
STATE OF CHARGE AND PARAMETER ESTIMATION OF ELECTRIC VEHICLE BATTERIES
}

\author{
Richard Bustos*, Abu Raihan Mohammad Siddique, \\ and Taranjit Cheema \\ College of Engineering and Physical Sciences, \\ University of Guelph, Ontario, Canada \\ *rbustos@uoguelph.ca
}

\begin{abstract}
Due to rising global environmental issues, electric vehicles (EV) are growing in popularity and will eventually replace vehicles that use internal combustion engines (ICE). EVs draw their power from batteries. Batteries are highly nonlinear storage elements used in a constantly changing environment making them highly dynamic and mathematically complex. In order to approximate the driving range of an EV, the state of charge (SOC) of the battery, which cannot be directly measured, has to be estimated accurately. SOC is highly dependent on the following parameters: internal resistance, temperature, and open circuit voltage. In this paper, two battery equivalent circuit models (ECM) are analyzed in conjunction with a thermal model to track the inner temperature of the battery. The states of the battery are estimated using the popular Kalman filter (KF) and unscented Kalman filter (UKF), and the results are discussed.
\end{abstract}

Keywords-electric vehicles; equivalent circuit models; Kalman filter; interactive multiple model; state of charge.

\section{INTRODUCTION}

The effects of technology, and in particular automobiles, on the environment are a growing concern in society. Automobile technology is rapidly moving towards a relatively eco-friendlier solution in an attempt to minimize the output of harmful greenhouse gasses: electric vehicles (EV) [1]. When compared to petroleum-based vehicles using the internal combustion engine (ICE), EVs have a smaller footprint, of course depending on the energy source [2].

In an EV, batteries store the electrical energy in an electrochemical reaction for later use. There are several types of batteries in the industry. The most popular are lead-acid, nickel, alkaline and lithium-ion [1]. Lithium has become very popular because it is the lightest of all metals, has the greatest electrochemical potential and provides the largest specific energy per weight [1]. Current lithium-ion battery technology allows EV to cover about $180-350 \mathrm{~km}$ per battery charge $[1,2]$. Unfortunately, batteries' full load capacity degrades over time as they are subject to charging cycles, resulting in a lower driving range throughout its lifetime [3]. In addition, the state of charge (SOC) and voltage measurement from the cell's terminal is crucial information to determine the available energy in the battery, which can be used to determine the

\author{
S. Andrew Gadsden ${ }^{\psi}$ and Shohel Mahmud \\ College of Engineering and Physical Sciences, \\ University of Guelph, Ontario, Canada \\ *gadsden@uoguelph.ca
}

available driving range of the EV. It is not possible to have direct measurements of the SOC [2]. The main difficulties are as follows:

- The battery packs of EVs have hundreds of cells connected in series; the different accumulated potential of each cell voltage is different to each other making it hard to have unified compensation or elimination methods.

- Voltage measurements require high precision. Other parameters are estimated according to voltage measurements. Required voltage precision is around 1 $\mathrm{mV}$ to have low carried \% errors [3].

The SOC of a battery can help estimate current driving range and prevent the battery pack from over charge and over discharge [3]. SOC is not easy to calculate for, as it would demand extensive computing times, expensive instrumentation or have the vehicle stopped preventing its application in real time [1, 4]. A solution is to generate estimates of the parameters, which requires a quality model, and good estimation strategy for the various scenarios: temperature, power demands, and state of function $[3,5]$.

In this paper, two battery electrical equivalent circuit models (ECM) are analyzed in conjunction with a thermal model. The states of the battery are estimated using the Kalman filter (KF) and unscented Kalman filter (UKF) [6, 7]. In addition, an interacting multiple model (IMM) is implemented for fault detection. Section II presents different filter techniques for the state of charge estimation process. Section III shows equivalent model of thermal, Rint, and Thevenin model. A numerical study and the simulated results are illustrated in Section IV. The paper is concluded in Section V.

\section{State OF Charge Estimation}

Three different estimation strategies, the Kalman filter $(\mathrm{KF})$, unscented Kalman filter (UKF) and the interacting multiple model (IMM) method, are used to estimate the SOC, terminal voltage, and temperature behaviour of the battery. The following section briefly summarizes the filters and their algorithms. 


\section{A. Kalman Filter $(K F)$}

The Kalman filter (KF) was first introduced by R.E. Kalman in 1960 [8]. The KF provides the optimal solution, in terms of state estimation error, for known linear systems in the presence of white, Gaussian noise [8]. The system dynamic model and measurement model is described by the following two equations, respectively [6-8]:

$$
\begin{gathered}
x_{k+1}=A_{k} x_{k}+B_{k} u_{k}+w_{k} \\
z_{k+1}=C_{k+1} x_{k+1}+v_{k+1}
\end{gathered}
$$

where $A$ is the system matrix, $B$ is the input matrix, $C$ is the output matrix, $x$ is the system states, $z$ is the measurement output, $u$ is the input, $w$ is the system noise, and $v$ is the measurement noise. The following equations represent the KF estimation process [6].

Prediction stage:

$$
\begin{aligned}
& \hat{x}_{k+1 \mid k}=A_{k} \hat{x}_{k \mid k}+B_{k} u_{k} \\
& P_{k+1 \mid k}=A_{k} P_{k \mid k} A_{k}^{T}+Q_{k}
\end{aligned}
$$

Update stage:

$$
\begin{gathered}
K_{k+1}=P_{k+1 \mid k} C_{k+1}^{T}\left[C_{k+1} P_{k+1 \mid k} C_{k+1}^{T}+R_{k+1}\right]^{-1} \\
\hat{x}_{k+1 \mid k+1}=\hat{x}_{k+1 \mid k}+K_{k+1}\left[z_{k+1}-C_{k+1} \hat{x}_{k+1 \mid k}\right] \\
P_{k+1 \mid k+1}=\left[1-K_{k+1} C_{k+1}\right] P_{k+1 \mid k}
\end{gathered}
$$

\section{B. Unscented Kalman Filter (UKF)}

The UKF is a form of sigma point Kalman filter (SPKF) which is based on a statistical linear regression strategy which linearizes the nonlinear model statistically [7]. In simple steps, SPKF techniques generate a certain number of points referred as 'sigma points' from the projected probability distribution of the states. These points are then projected using the nonlinear system model, to obtain the 'a posteriori' estimate for probability distribution. The main difference characteristic of the SPKF is that it does not calculate the Jacobian matrices of the system in an attempt to linearize the system often yielding better results than other forms of the KF such as the Extended Kalman Filter (EKF). In the literature, one of the most popular type of SPKF is the UKF.

The following is a summary of the UKF algorithm [8]:

Intialization:

$$
\begin{gathered}
X_{0, k \mid k}=\hat{x}_{k \mid k} \\
W_{0}=\frac{k}{(n+k)} \\
\hat{x}_{k \mid k}+\left(\sqrt{(n+k) P_{k \mid k}}\right)_{i} \\
W_{i}=\frac{1}{[2(n+k)]} \\
X_{i+n, k \mid k}=\hat{x}_{k \mid k}-\left(\sqrt{(n+k) P_{k \mid k}}\right)_{i}
\end{gathered}
$$

Prediction:

$$
W_{i}=\frac{1}{[2(n+k)]}
$$

$$
\begin{aligned}
\hat{X}_{i, k+1 \mid k} & =f\left(X_{i}, u_{k}, w_{k}\right) \\
\hat{x}_{k+1 \mid k} & =\sum_{i=0}^{2 n} W_{i, k \mid k}, u_{k} \\
P_{k+1 \mid k}=\sum_{i=0}^{2 n} W_{i}\left(\hat{X}_{i, k+1 \mid k}\right. & \left.-\hat{x}_{k+1 \mid k}\right)\left(\hat{X}_{i, k+1 \mid k}-\hat{x}_{k+1 \mid k}\right)^{T} \\
\hat{Z}_{i, k+1 \mid k} & =h\left(\hat{X}_{i, k+1 \mid k}, u_{k}\right) \\
\hat{Z}_{k+1 \mid k} & =\sum_{i=0}^{2 n} W_{i} Z_{i, k+1 \mid k}
\end{aligned}
$$

Update:

$$
\begin{gathered}
P_{z z . k+1 \mid k}=\sum_{i=0}^{2 n} W_{i}\left(\hat{Z}_{i, k+1 \mid k}-\hat{z}_{k+1 \mid k}\right)\left(\hat{Z}_{i, k+1 \mid k}-\hat{z}_{k+1 \mid k}\right)^{T} \\
P_{x z . k+1 \mid k}=\sum_{i=0}^{2 n} W_{i}\left(\hat{X}_{i, k+1 \mid k}-\hat{x}_{k+1 \mid k}\right)\left(\hat{Z}_{i, k+1 \mid k}-\hat{z}_{k+1 \mid k}\right)^{T} \\
K_{k+1}=P_{x z \cdot k+1 \mid k} P_{z z \cdot k \mid k}^{-1} \\
\hat{x}_{k+1 \mid k+1}=\hat{x}_{k+1 \mid k}+K_{k+1}\left(z_{k+1}-\hat{z}_{k+1 \mid k}\right) \\
P_{k+1 \mid k+1}=P_{k+1 \mid k}-K_{k+1} P_{z z, k+1 \mid k} K_{k+1}^{T}
\end{gathered}
$$

\section{Interacting Multiple Mode (IMM)}

The interacting multiple model (IMM) strategy is useful when a system behaves according to more than one mode of operation or regime. Most engineering systems perform according to a number of different models. The IMM utilizes a finite number of models that calculates likelihood values based on the state estimates and state error covariance. These likelihood values are used to determine operating mode probabilities, and can be used for fault detection $[6,9]$.

A summary of the IMM algorithm broken down into 5 steps is shown below [8]:

Calculation of the mixing probablities:

$$
\begin{gathered}
\mu_{i|j, k| k}=\frac{1}{\overline{c_{j}}} p_{i j} \mu_{i, k} \\
\overline{c_{j}}=\sum_{i=1}^{r} p_{i j} \mu_{i, k}
\end{gathered}
$$

Mixing stage:

$$
\begin{gathered}
\hat{x}_{0 j, k \mid k}=\sum_{i=1}^{r} \hat{x}_{i, k \mid k} \mu_{i|j, k| k} \\
P_{0 j, k \mid k}=\sum_{i=1}^{r} \mu_{i|j, k| k}\left\{P_{i, k \mid k}+\left(\hat{x}_{i, k \mid k}-\hat{x}_{0 j, k \mid k}\right)\left(\hat{x}_{i, k \mid k}-\hat{x}_{0 j, k \mid k}\right)^{T}\right\} \\
\text { Mode - matched filtering: } \\
\Lambda_{j, k+1}=\mathcal{N}\left(z_{k+1} ; \hat{z}_{j, k+1 \mid k}, S_{j, k+1}\right) \\
\Lambda_{j, k+1}=\frac{1}{\sqrt{\left|2 \pi S_{j, k+1}\right|_{A b s}}} \exp \left(\frac{-\frac{1}{2} e_{j, z, k+1}^{T} e_{j, z, k+1 \mid k}}{S_{j, k+1}}\right)
\end{gathered}
$$

Mode probability update:

$$
\mu_{j, k}=\frac{1}{c} \Lambda_{j, k+1} \sum_{i=1}^{r} p_{i j} \mu_{i, k}
$$




$$
c=\sum_{j=1}^{r} \Lambda_{j, k+1} \sum_{i=1}^{r} p_{i j} \mu_{i, k}
$$

State estimate and covariance combination:

$$
\begin{aligned}
\hat{x}_{k+1 \mid k+1} & =\sum_{j=1}^{r} \mu_{j, k+1} \hat{x}_{j, k+1 \mid k+1} \\
P_{k+1 \mid k+1}=\sum_{j=1}^{r} \mu_{j, k+1} & \left\{P_{j, k+1 \mid k+1}\right. \\
+ & \left(\hat{x}_{j, k+1 \mid k+1}\right. \\
- & \left.\left.\hat{x}_{k+1 \mid k+1}\right)\left(\hat{x}_{j, k+1 \mid k+1}-\hat{x}_{k+1 \mid k+1}\right)^{T}\right\}
\end{aligned}
$$

III. Electrical Equivalent Circuit Models

ECM are models based on electrical components such as ideal voltage sources, resistors, and capacitors to simulate the behaviour of the battery. The vast majority of ECM models are semi-empirical models where the process of calculating your final outputs is based on two distinctive activities. First, the OCV with respect to SOC must be known beforehand by performing laboratory experiments where the battery is put through discharge and resting cycles. Second, the ECM parameters such as resistors and capacitors must be calibrated through I/O data using parameter identification techniques such as least square methods. ECM model design may be easily achieved, but depending on the output and accuracy, they can vary greatly [1]. Moreover, computational power and the simulation time needed to simulate the battery is low. These characteristics make ECM models more suitable for use and implementation in a battery management system (BMS) [12].

\section{A. Thermal Model}

Battery thermal management is critical for tracking and estimating parameters, as well as avoiding severe consequences. The thermal model used for determining the core temperature, $T_{c}$, and the surface temperature of the cell, $T_{S}$, is shown in Figure 1, where the core and the surface of the cell are modeled as RC branches [13]. The core's resistance, $R_{c}$, models the amount of heat flux leaving or entering the core and the capacitor, $C_{c}$, models the core's ability to store heat. The surface of the battery was modeled similarly, yielding four parameters. The two inputs to the model are demanded/supplied current, $I_{s}$, and forced convection to the skin of the cell, $T_{f}$.

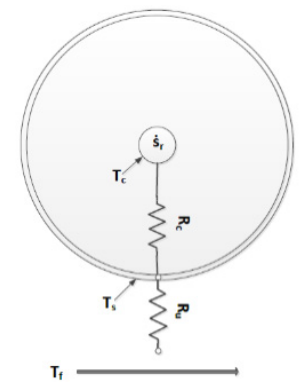

Figure 1. Schematic diagram of the battery thermal model [13].

The two differential equations for a cylinder cell were previously analyzed by [14] and [4]:

$$
\dot{T}_{c}=\frac{1}{C_{c}}\left(\dot{S}_{c}+\frac{\dot{T}_{s}-T_{c}}{R_{c}}\right)
$$

$$
\dot{T}_{c}=\frac{1}{C_{s}}\left(\frac{T_{f}-T_{s}}{R_{u}}-\frac{T_{s}-T_{c}}{R_{c}}\right)
$$

where $\dot{s}_{r}=\left(O C V-V_{T}\right) I_{s}, \mathrm{OCV}$ is the open circuit voltage, $V_{T}$ is the terminal voltage. The following is the state space representation of the two differential equations:

$$
\begin{gathered}
A=\left(\begin{array}{cc}
-\frac{1}{R_{c} C_{c}} & \frac{1}{R_{c} C_{c}} \\
-\frac{1}{R_{c} C_{s}} & -\frac{1}{C_{s}}\left(\frac{1}{R_{c}}+\frac{1}{R_{s}}\right)
\end{array}\right) \\
B=\left(\begin{array}{cc}
\frac{O C V-V_{t}}{C_{c}} & 0 \\
0 & -\frac{1}{C_{s} R_{s}}
\end{array}\right) \\
u=\left[\begin{array}{ll}
I_{s} & T_{f}
\end{array}\right]^{\prime}
\end{gathered}
$$

This thermal model was used across all simulated ECMs. Furthermore, a value of $25^{\circ} \mathrm{C}$ was used for $T_{f}$ to simulate the battery inside a laboratory with the air conditioner on. The values for the model's parameters were taken from [13] and used to generate the simulation results.

\section{B. Rint Model}

The most basic and commonly used battery model is the Rint model. It consists of an ideal battery with an open-circuit voltage $U_{O C}$ and a constant equivalent internal series resistance $R_{O} . U_{L}$ is the terminal voltage of the battery and $I_{L}$ is the load current with a negative value at charging and a positive value at discharging:

$$
U_{L}=U_{O C}-R_{O}
$$

The equations for the state space representation of the Rint model are as follows:

$$
\begin{gathered}
x=\left[S O C, T_{\text {core }}, T_{\text {surface }}\right]^{\prime} \\
A=\left(\begin{array}{cc}
1 & 0 \\
0 & A_{\text {thermal }}
\end{array}\right) \\
B=\left(\begin{array}{ll}
\frac{1}{C_{\text {battery }}} & 0 \\
0 & B_{\text {thermal }}
\end{array}\right) \\
u=\left[\begin{array}{ll}
I_{s} & T_{f}
\end{array}\right]^{\prime}
\end{gathered}
$$


The state space representation of the system was discretized for implementation purposes. Once the SOC was derived based on the state space, the algorithm would update the parameter values for that SOC level and calculate the corresponding $V_{O C}$. The terminal voltage $\left(V_{T}\right)$ was derived using equation (13). The following section describes the corresponding output of the model based on Normal Conditions.

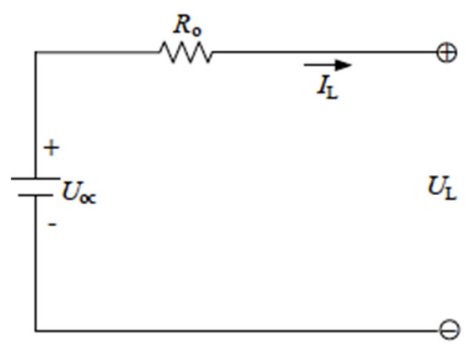

Figure 2. Schematic circuit diagram of Rint Model [7].

\section{Thevenin Model}

The Thevenin model is often referred to as a first order battery model. It consists of a parallel RC circuit in series with a resistance. As shown in Figure 3, it is mainly composed of three parts: open-circuit voltage source $\left(U_{O C}\right)$, internal resistance, and equivalent capacitances. The internal resistances include the ohmic resistance $\left(R_{O}\right)$ connected in series and the polarization resistance $\left(R_{T h}\right)$ connected in parallel. Polarization resistance is used so that the battery does not provide energy (or 'drain') right away to the system. The equivalent capacitance $\left(C_{T h}\right)$ is used to describe the transient response during charging and discharging. $\left(U_{T h}\right)$ is the Thevenin voltage across the $\left(C_{T h}\right),\left(I_{T h}\right)$ is the outflow current of $\left(C_{T h}\right)$ and $\left(U_{T h}\right)$ is the terminal voltage. The electrical behavior (dynamics) of the Thevenin model can be expressed by Eq. 18 [11].

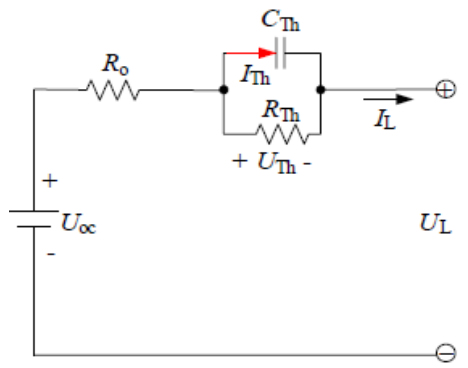

Figure 3. Schematic circuit diagram of Thevenin Model [11].

$$
\begin{gathered}
\dot{U}_{T h}=-\frac{U_{T h}}{R_{T h} C_{T h}}+\frac{I_{L}}{C_{T h}} \\
U_{L}=U_{O C}-U_{T h}-I_{L} R_{o}
\end{gathered}
$$

\section{NUMERICAL SimUlation AND RESUltS}

Figure 4 depicts the supplied current used across the different models in this numerical study. Positive current was assumed to demand from the battery, thus discharging the battery. The algorithm to simulate the battery was developed in MATLAB ${ }^{R}$. The SOC was estimated based on the following equation:

$$
S O C=S O C_{o}+\frac{1}{C_{\text {battery }}} \int_{0}^{t} I_{s} d t
$$

where the $C_{\text {battery }}$ refers to the nominal cell or battery capacity.

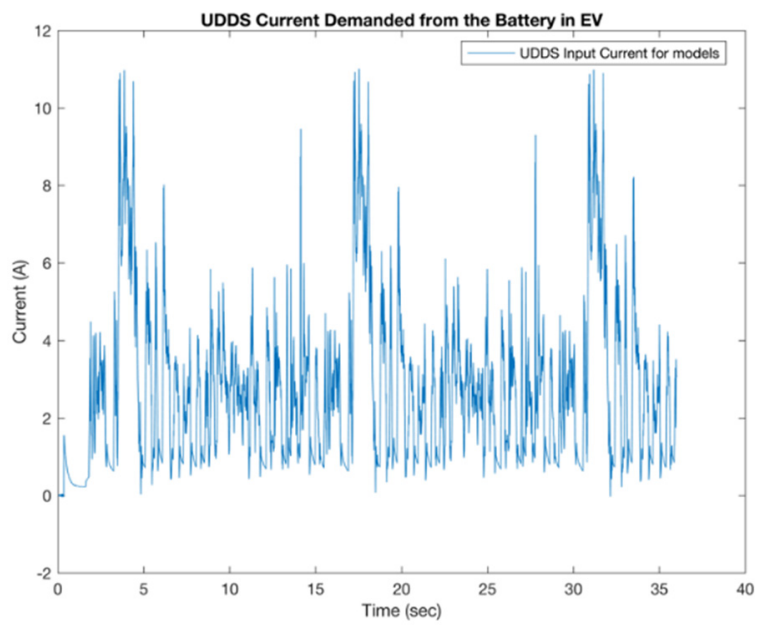

Figure 4. UDDS current profile.

As mentioned earlier, due to their dependence on SOC levels, model parameters curves are often derived offline experimentally using constant current (CC), and constant voltage $(\mathrm{CV})$ charge and discharge cycles under constant temperature by using a thermal chamber. Similar experiments are performed at different temperatures and lookup tables are created for the models. Afterwards, the data is processed using software such as MATLAB to match parameters to a desired transfer function [15]. Other parameter identification techniques use genetic algorithms (GA) [14].

In this paper, the MATLAB ${ }^{\circledR}$ Simulink model was obtained from [17] and modified for the corresponding models to optimize the values of the model parameters: $U_{O C}, R_{O}, R_{T h}$, and $C_{T h}$. Furthermore, the input current and output voltage shown on Figure 5 were used for optimizing the parameter values.
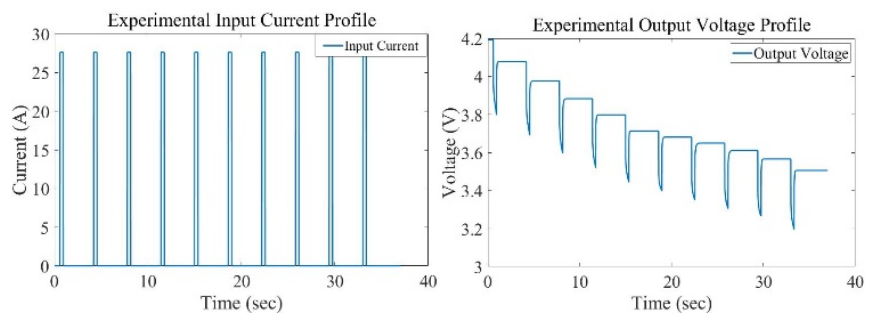

Figure 5. Input current Profile used across all models.

Figure 6 illustrates the resulting VOC-SOC curve from the optimization process. The VOC gradually increases with increasing SOC and reaches maximum at $4.2 \mathrm{~V}$. This VOCSOC curve was used for both models. Figure 7 shows the resulting capacitance values for the Thevenin capacitance, $C_{t h}$ for all levels of SOC. The nonlinearity of $C_{t h}$ is inherited by the model; making it nonlinear and thus, resulting in the implementation of the UKF method. Figure 8 depicts the calibration results of the Simulink model using the inputoutput data shown in Figure 5. 
Lastly, the system and measurement noise covariance matrices were set to $Q=10^{-9}$ eye(4) and $R=$ $\operatorname{diag}\left(\left[\begin{array}{lll}10^{-4} & 0.00172 & 10^{-4}\end{array}\right]\right)$. The initial state error covariance was set to $P=10 Q$ and the $C_{\text {battery }}$ was 27.625 Ah.

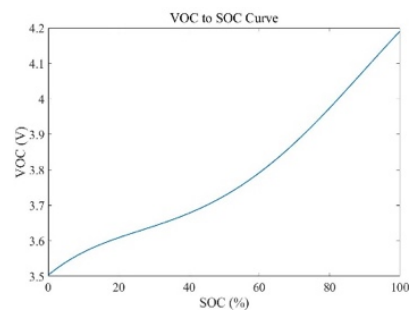

Figure 6. Open circuit voltage with respect to percentage of SOC for Rint model.

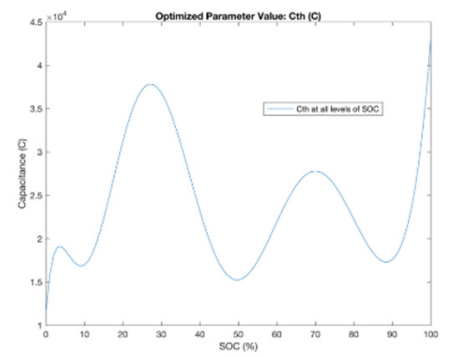

Figure 7. $C_{t h}$ profile with respect to percentage of SOC for Thevenin model.
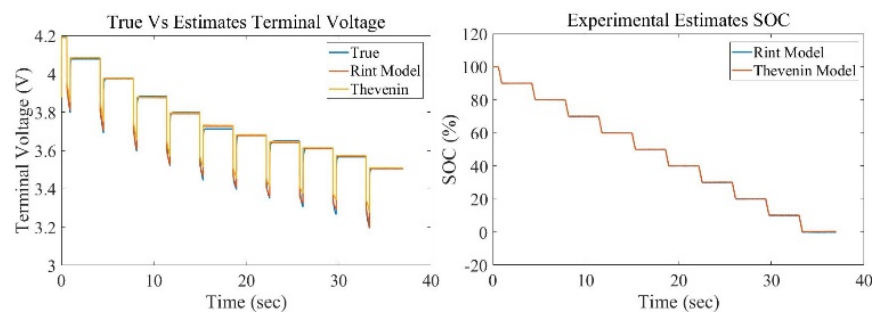

Figure 8. Calibrated Results of (a) terminal volatge and (b) SOC between Rint and Thevenin model.

\section{A. Rint Model}

The terminal voltage and the percentage of SOC for KF and IMM-KF of Rint model is presented in Fig. 10(a) and 10(b), respectively. Both terminal voltage and percentage of SOC decrease as time passes and $\mathrm{KF}$ almost follows the true value. However, as before, the IMM-KF tries to follow the trajectory but is slightly off from the true value. From Fig. 10(b), the KF follows the true SOC value and the IMM-KF is relatively close.

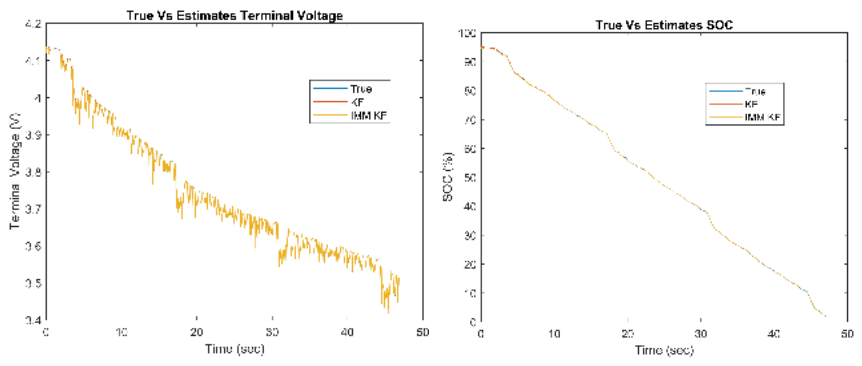

Figure 9. Simulated results of Rint model. (a) Terminal volatge and (b) SOC along with time for KF and IMM KF.
Using Thermal model, the temperature effect for Rint model is also analyzed. Figure 11(a) and 11(b) show the core and surface temperature behavior with respect to time of Rint model, respectively. Both temperatures decrease with time. Moreover, the surface temperature exponentially deceases and approaches $6{ }^{\circ} \mathrm{C}$ whereas the core temperature goes to nearly $23^{\circ} \mathrm{C}$. This is due to the thermal resistance between the core and surface, which is higher than the resistance between the surface and the air (e.g., natural cooling effect).
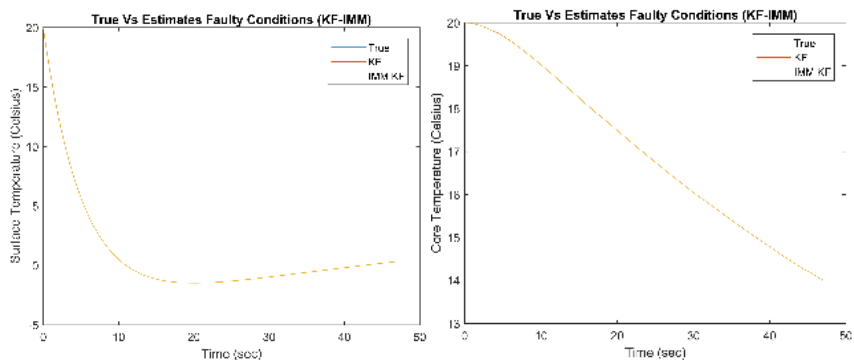

Figure 10. Simulated results of (a) core and (b) surface temperature for the KF and IMM-KF (Rint model).

\section{B. Thevenin Model}

The terminal voltage and SOC as a function of time are illustrated in Fig. 9(a) and 9(b), respectively. Terminal voltage shows the comparison between true values, UKF and IMMUKF. It can be clearly seen from Fig. 9(a) that with time, the terminal voltage decreases and UKF follows the true value. Whereas the IMM-UKF is a slightly off from the true value. In this scenario, the UKF obtains the best result. A similar trend can be identified from the SOC curve in Fig. 9(b).
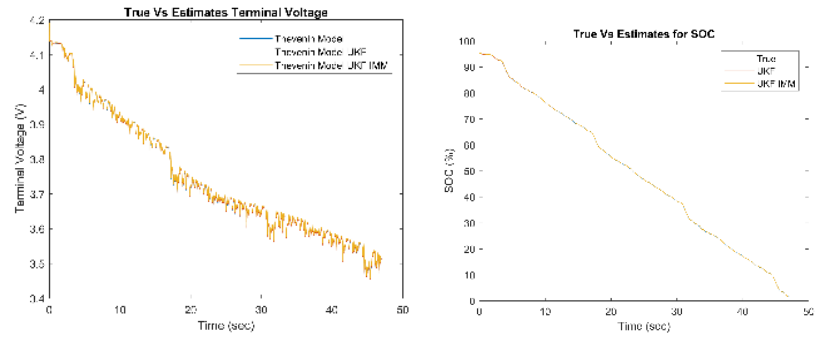

Figure 11. Simulated results of Thevenin model. (a) Terminal volatge and (b) SOC for the KF and IMM-KF.

\section{CONCLUSIONS}

An analysis on two ECM battery models, as well as a popular thermal model, was presented in this paper. Moreover, states such as $S O C, V_{T}, T_{c}$, and $T_{s}$ of the battery were estimated using the KF, UKF and IMM-KF estimation strategies. Parameter identification was performed using a modified MATLAB ${ }^{\circledR}$ Simulink model to calibrate battery models to a corresponding set of input-output data. Finally, the IMM was implemented for successful fault detection were the battery was aged and the thermal core resistance parameter was increased. Future work involves the development of an experimental testbed and implementation and comparison of other nonlinear battery models. 


\section{REFERENCES}

[1] A. Davide, Battery Management System for large Lithium-Ion Battery Packs, Norwood: Artech House, 2010.

[2] Z. Y. Hou, P. Y. Lou and C. C. Wang, "State of Charge, State of Health, and State of Function Monitoring for EV BMS," in IEEE International Conference on Consumer Electronics, 2017.

[3] L. Lu, X. Han, J. Li, J. Hua and M. Ouyang, "A review on the key issues for lithium-ion battery management in electric vehicles," Journal of Power Sources, vol. 226, pp. 272-288, 2013.

[4] Y. Masoudi, "Real-time Optimal Battery Thermal Management System Controller for Electric and Plug-in Hybrid electric Vehicles," University of Waterloo, Waterloo, 2017.

[5] A. Millner, "Modeling Lithium Ion battery degradation in electric vehicles," in IEEE Conference on Innovative Technologies for an Efficient and Reliable Electricity Supply, 2010.

[6] S. A. Gadsden, "Smooth Variable Structure Filtering: Theory and Applications," Ph.D. Thesis, McMaster University, Hamilton, Ontario, 2011.

[7] E. A. Wan and R. van der Merwe, "The Unscented Kalman Filter for Nonlinear Estimation," Oregon Graduate Institute of Science \& Technology, Beaverton

[8] S. A. Gadsden, "Smooth Variable Structure Filtering: Theory and Applications," Hamilton, 2011

[9] S. A. Gadsden and A. S. Lee, "Advances of the Smooth Variable Structure Filter: Square-Root and Two-Pass Formulations," Journal of Applied Remote Sensing, vol. 11, no. 1, pp. 1-19, 2017.
[10] M. Farag, "Lithium-Ion Batteries Modelling and State of Charge Estimation", Hamilton: McMaster University, 2013

[11] H. He, R. Xiong and J. Fan, "Evaluation of Lithium-Ion Battery Equivalent Circuit Models for state of charge estimation by an experimental Approach," Energies, vol. 4, pp. 582 - 598, 2011.

[12] A. Nikolian et al., "Classsification of Electric Modelling and Characterization Methods of Lithium-ion Mierlo," in EUR.ELCTR.VEH.CONGR, 2014.

[13] I. Arasaratnam, J. Tjong, R. Ahmed, M. El-Sayed and S. Habibi, "Adaptive Temperature Monitoring for Battery Thermal Management," in Transportation Electrification Conference and Expo (ITEC), 2013 IEEE, Detroit, 2013.

[14] C. Park and A. Jaura, "Dynamic Thermal Model of Li- Ion Battery for Predictive Behavior in Hybrid and Fuel Cell Vehicles," in 2003 Future Transportation Technology Conference : SAE technical paper, Warrendale,, 2003.

[15] S. Zhao and D. . A. Howey, "Global sensitivity analysis of battery equivalent circuit model parameters," niversity of Oxford, Oxford, 2016.

[16] V. Sangwan, A. Sharma, R. Kumar and A. . K. Rathore, "Estimation of Optimal Li-ion Battery Parameters considering c-rate, SOC and Temperature," IEEE, Singapore, 2016.

[17] J. Gazzarri, "MathWorks" $10 \quad 08$ 2017.[Online]. Available: https://www.mathworks.com/matlabcentral/fileexchange/36019-lithiumbattery-model-simscape-language-and-simulink-design-optimization. [Accessed 0602 2018] IEEE, Singapore, 2016. 